\title{
Gas-phase induced Marangoni flow causes unstable drop merging
}

\section{Supplementary materials}

Peyman Rostami ${ }^{1,2 * *}$, Benedikt B. Straub ${ }^{l}$ Günter K. Auernhammer ${ }^{1,2, *}$

${ }^{1}$ Leibniz Institute for Polymer Research, Hohe Straße, 01069 Dresden, Germany

${ }^{2}$ Max Planck Institute for Polymer Research, Ackermannweg 10, 55128 Mainz, Germany

Email:

*auernhammer@ipfdd.de

*rostami@ipfdd.de

Movie 1: The coalescence between and an already deposited water drop (upper droplet) and Cyclohexyl bromide drop (Fig. S1).

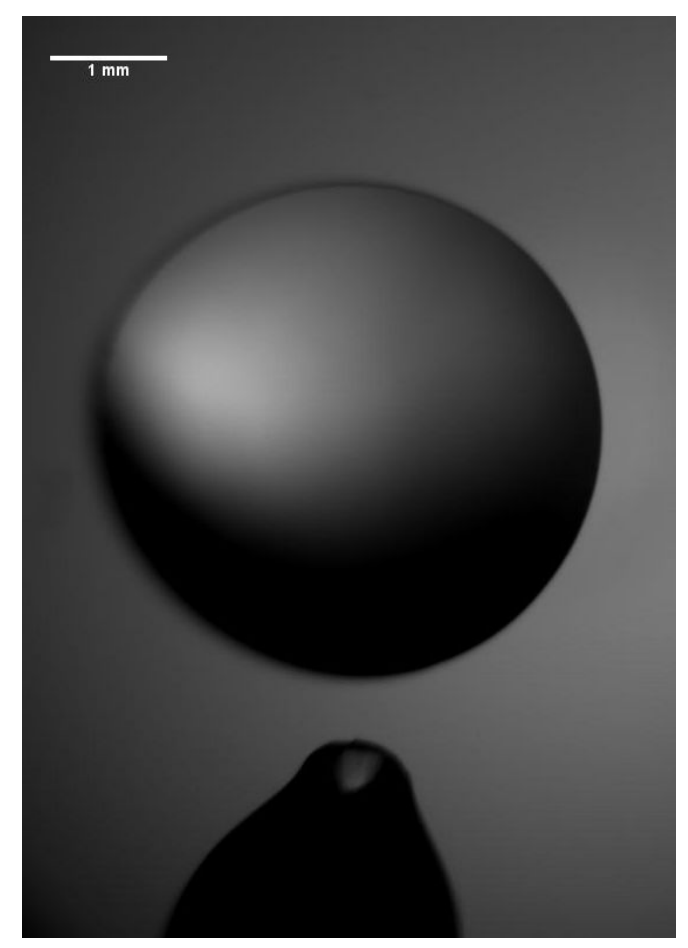

Fig. S1: Coalescence of water drop with Cyclohexyl bromide drop 
Movie 2: The coalescence between and already deposited oil drop (left) and the mixture of water and isopropanol (50 w/w) (Fig. S2).

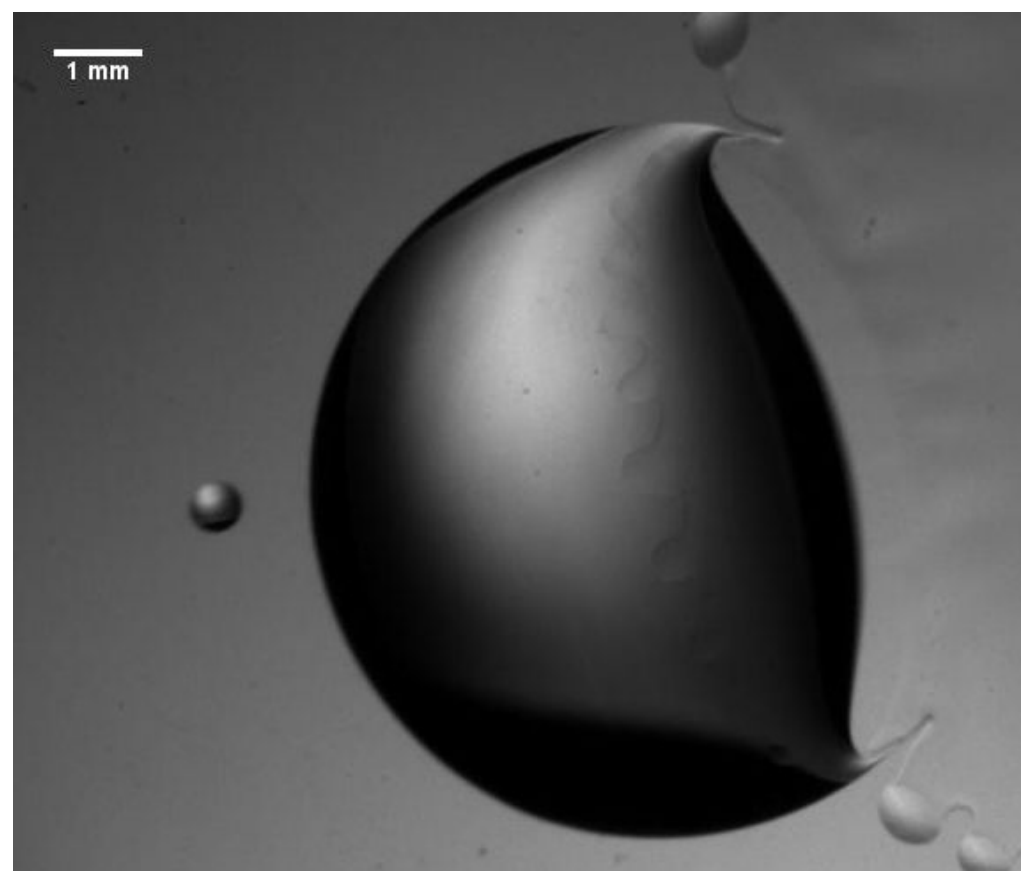

Fig. S2: Coalescence of oil drop with mixture of water and IPA drop.

Movie 3: Induced flow by solvo-capillary effect inside the water drop (right drop) due to presence of cyclohexyl bromide drop in nearby (on left) (Fig. S3) .

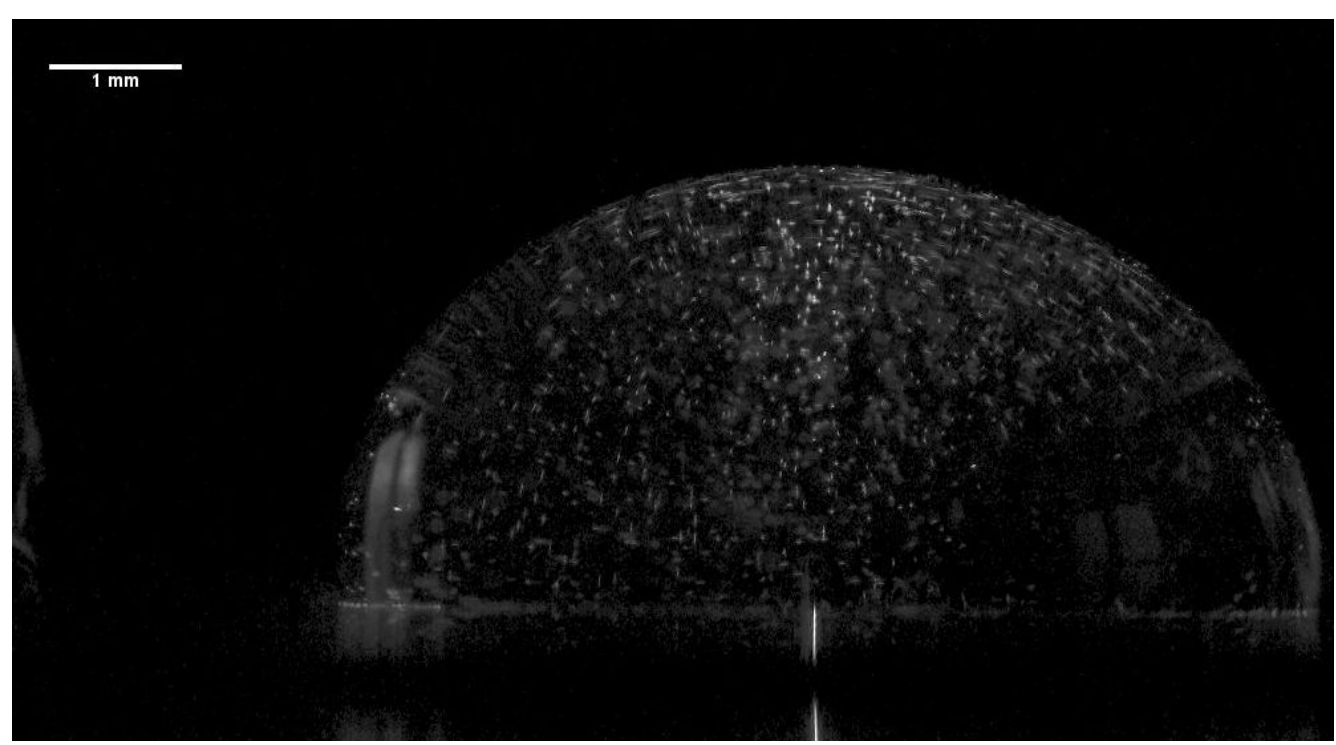

Fig. S3: The already deposited water drop (right) and cyclohexyl bromide drop in left, which induces flow inside water drop. 


\section{Spreading velocity:}

Spreading velocity of second drop is the velocity of contact line of second drop, which for a constant volume it decays over time and become zero after full spreading (Fig.S4).

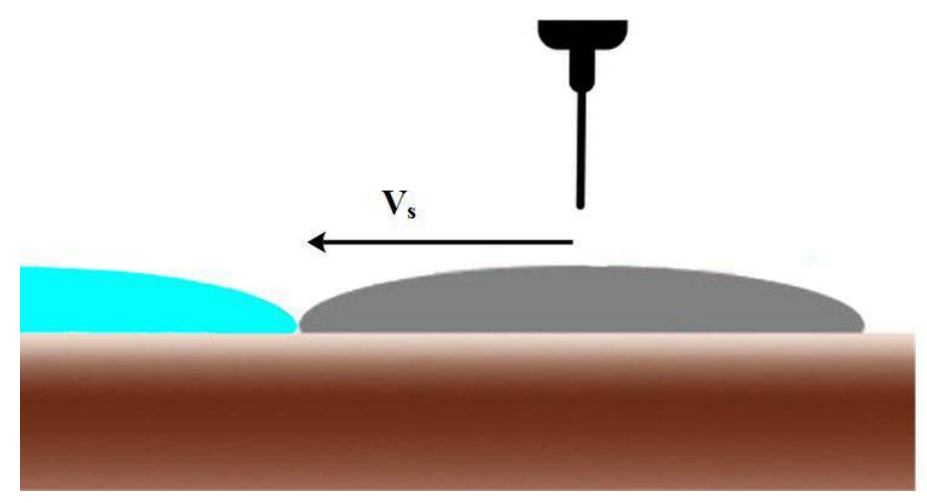

Fig. S4: Schematic of injection process and spreading velocity.

\section{Lab condition:}

All the experiments were performed in lab condition, Fig. S5 shows the temperature and relative humidity of the lab for one day and it is monitored every day and shows same behavior.

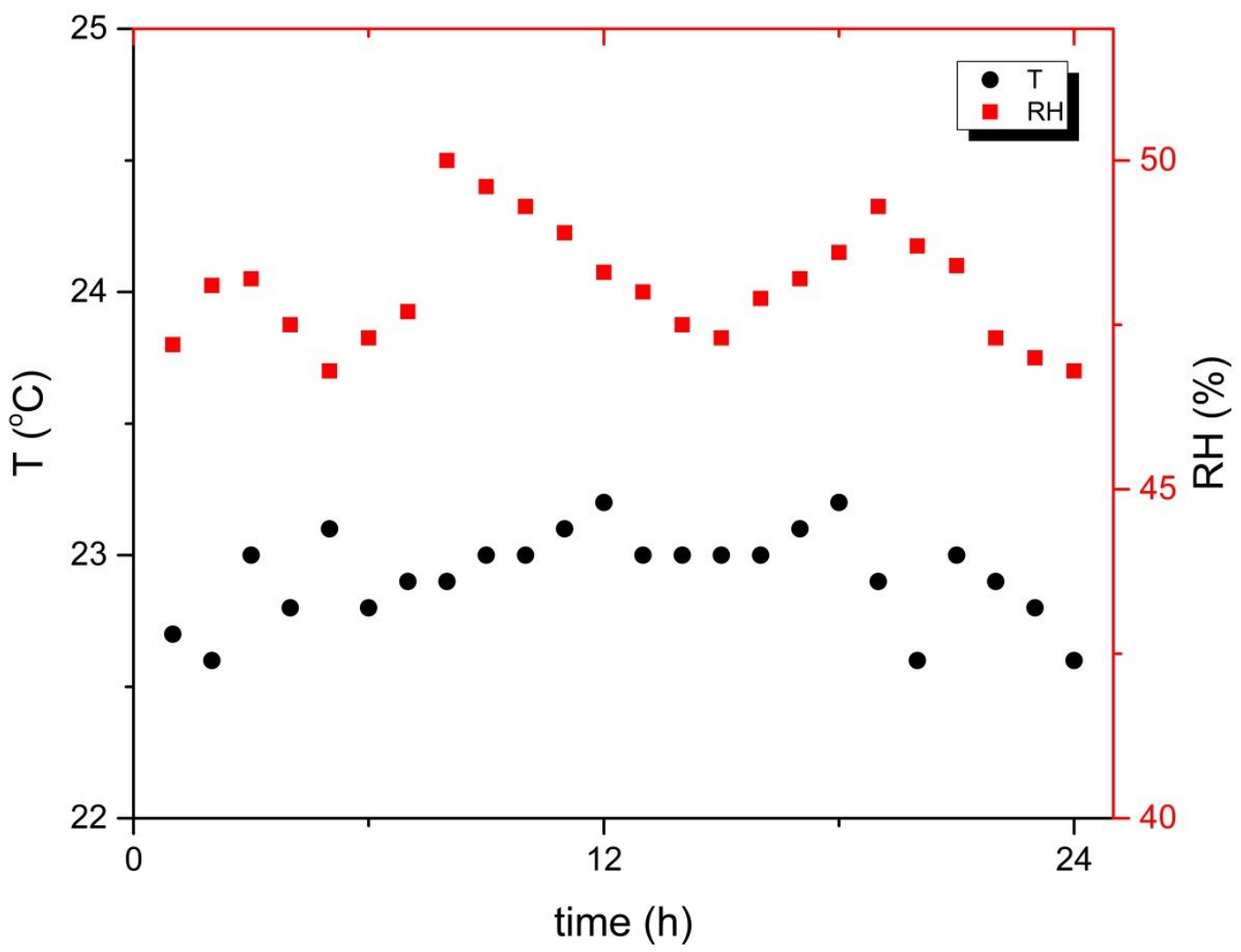

Fig. S5: Lab temperature and relative humidity for one day. 